\title{
Modified multivariate F-transform based on B-splines
}

\author{
Martins Kokainis ${ }^{a}$ and Svetlana Asmuss ${ }^{a, b}$ \\ ${ }^{a}$ University of Latvia, Department of Mathematics, \\ Jelgavas 3, Riga, LV-1004, Latvia, martins.kokainis@lu.lv \\ ${ }^{b}$ Institute of Mathematics and Computer Science, University of Latvia, \\ Raina bulvaris 29, Riga, LV-1459, Latvia, svetlana.asmuss@lu.lv
}

\begin{abstract}
We deal with the higher degree multivariate fuzzy transforms ( $F$-transforms with polynomial components) in the case when generalized fuzzy partition of the Cartesian product of intervals is given by multivariate Bsplines. The aim of this paper is to generalize the technique of modified $F$-transform introduced previously for the univariate case to the multivariate case. The proposed modification allows to extend good approximation properties of the multivariate spline-based $F$ transform from the subset where the Ruspini condition is fulfilled to the whole initial domain.
\end{abstract}

Keywords: Multivariate fuzzy transform, B-spline, extrapolation, approximation error

\section{Introduction}

The technique of ordinary $F$-transforms introduced by I. Perfilieva $[13,10]$ was generalized to the case of higher degree (i.e., $F^{m}$-transforms) in [12] as well as extended to the multivariate version (see, e.g., $[16,14,3,5])$. In all these settings, properties of $F$-transforms significantly depend on basic functions which form a fuzzy partition. We focus on $F^{m}$ transforms with respect to a spline-based fuzzy partition proposed in [6], generalized for the multivariate case in [7].

Previously we have proved that using B-splines may improve approximation properties of $F^{m}$-transforms for smooth functions of one and also of many variables However, the approximation error estimations both in the univariate case [8] and in the multivariate case [7] are true only in the subset where the Ruspini condition is fulfilled. To eliminate this obstacle for the effective use of the advantages of the $F^{m}$-transform technique in applications a modification of the spline-based $F^{m}$ transform was proposed. It was done by the authors for the univariate case at the IPMU 2018 conference $[9]$.

The aim of this paper is to generalize the modification introduced in [9] to the multivariate case. As in the univariate case, the modification is done by adding more basic functions to the fuzzy partition and by applying an extrapolation technique with respect to functions under consideration. The paper is organized as follows. Section 2 contains preliminaries on splinebased fuzzy partitions and $F^{m}$-transforms (direct, inverse and composite). Section 3 describes the modification technique including extension of spline-based fuzzy partition and extrapolation technique. Section 4 is devoted to the investigation of approximation properties of the modified spline-based $F^{m}$-transforms for smooth functions.

\section{Preliminaries}

\section{$2.1 \quad$ Notation in $\mathbb{R}^{d}$}

Let $\mathbb{N}$ be the set of positive integers and $\mathbb{N}_{0}=\mathbb{N} \cup\{0\}$ be the set of nonnegative integers. Let $d \in \mathbb{N}$ be a fixed space dimension. The elements of the set $\mathbb{N}_{0}^{d}$ are called multi-indices. For a multi-index $\mathbf{j}=\left(j_{1}, \ldots, j_{d}\right) \in \mathbb{N}_{0}^{d}$ we denote $|\mathbf{j}|=j_{1}+j_{2}+\ldots+j_{d}$. The multi-indices can be added and subtracted in the usual way: $\mathbf{k} \pm \mathbf{j}=$ $\left(k_{1} \pm j_{1}, k_{2} \pm j_{2}, \ldots, k_{d} \pm j_{d}\right)$; moreover, we write $\mathbf{k}<\mathbf{j}$ if simultaneously $k_{1}<j_{1}, \ldots, k_{d}<j_{d}$. Furthermore, if $c \in \mathbb{R}$ is a scalar, then $c \mathbf{j}$ stands for $\left(c j_{1}, \ldots, c j_{d}\right)$ and $c<\mathbf{j}$ means that simultaneously $c<j_{1}, \ldots, c<j_{d}$. The factorial of a multi-index $\mathbf{j}=\left(j_{1}, \ldots, j_{d}\right)$ is defined as $\mathbf{j} !=j_{1} ! j_{2} ! \ldots j_{d} !$

For a multi-index $\alpha=\left(\alpha_{1}, \ldots, \alpha_{d}\right)$ the higher order

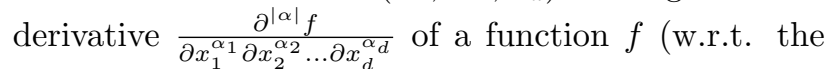
variable $\left.\mathbf{x}=\left(x_{1}, \ldots, x_{d}\right)\right)$ is denoted by $\partial^{\alpha} f$.

For a vector of $d$ real variables $\mathbf{x}=\left(x_{1}, \ldots, x_{d}\right) \in$ 
$\mathbb{R}^{d}$ and a multi-index $\mathbf{l}=\left(l_{1}, \ldots, l_{d}\right) \in \mathbb{N}_{0}^{d}$ we denote the monomial $x_{1}^{l_{1}} x_{2}^{l_{2}} \ldots x_{d}^{l_{d}}$ by $\mathbf{x}^{\mathbf{l}}$. Then $\mathbf{l}$ is called the degree of the monomial $\mathbf{x}^{\mathbf{l}}$ and $|\mathbf{l}|$ is called its total degree. We denote by $\mathbb{P}_{m}^{d}$ the space of all polynomials in $d$ variables of the total degree at most $m \in \mathbb{N}_{0}$.

Finally, for $\mathbf{x} \in \mathbb{R}^{d}$ and a positive real $r$ we denote the respective $\ell^{\infty}$ ball as

$$
\mathfrak{B}_{r}(\mathbf{x})=\prod_{i=1}^{d}\left[x_{i}-r, x_{i}+r\right] .
$$

\subsection{Univariate uniform fuzzy partition}

Consider an interval $[a, b] \subset \mathbb{R}$. Let $N \in \mathbb{N}$ and $h>$ $0, h^{\prime}>h / 2$. Suppose that $t_{0}, \ldots, t_{N}$ are $h$-equidistant nodes s.t. $a<t_{0}<\ldots<t_{N}<b$. Furthermore, suppose that $\bigcup_{j=0}^{N}\left[t_{i}-h^{\prime}, t_{i}+h^{\prime}\right]=[a, b]$.

Definition 1 ([11]). Fuzzy sets $A_{0}, \ldots, A_{N} \in C[a, b]$ are said to constitute a generalized $\left(h, h^{\prime}\right)$-uniform fuzzy partition (FP for short) of $[a, b]$ if the following conditions are satisfied: for all $j \in\{0, \ldots, N\}$ :

- $A_{j}(t) \in(0,1]$ if $t \in\left(t_{j}-h^{\prime}, t_{j}+h^{\prime}\right)$, and $A_{j}(t)=0$ otherwise;

- $A_{j}\left(t_{j}-t\right)=A_{j}\left(t_{j}+t\right)$ for all $t \in\left[0, h^{\prime}\right]$;

- $\sum_{j=0}^{N} A_{j}(t)>0$ for all $t \in(a, b)$;

- $A_{j}(t)=A_{j+1}(t+h)$ for all $t \in\left[t_{j}-h^{\prime}, t_{j}+h^{\prime}\right]$, if $j \neq N$

Then there is a function $A:[-H, H] \rightarrow \mathbb{R}$ (called the generating function of the partition), where $H=h^{\prime} / h$, s.t. for all $j \in\{0, \ldots, N\}$ and $t \in\left[t_{j}-h^{\prime}, t_{j}+h^{\prime}\right]$, $A_{j}(t)=A\left(\frac{t-t_{j}}{h}\right)$.

Furthermore, if there is an interval $I \subset[a, b]$ such that $\sum_{j=0}^{N} A_{j}(t)=1$ for all $t \in I$, the fuzzy partition is said to fulfill the Ruspini condition on $I$.

\subsection{Multivariate fuzzy partition}

Let $\left[a_{i}, b_{i}\right] \subset \mathbb{R}$ for all $i \in\{1, \ldots, d\}$ and denote $I_{a b}=$ $\prod_{i=1}^{d}\left[a_{i}, b_{i}\right]$. Let $A_{0}^{(i)}, \ldots, A_{N_{i}}^{(i)}$ be a fuzzy partition of $\left[a_{i}, b_{i}\right]$ for all $i$, then the collection $\left\{A_{\mathbf{j}}: 0 \leq \mathbf{j} \leq \mathbf{N}\right\}$, $\mathbf{N}=\left(N_{1}, \ldots, N_{d}\right)$, is called a fuzzy partition (FP) of $I_{a b}$, where

$$
A_{\mathbf{j}}(\mathbf{x}):=\prod_{i=1}^{d} A_{j_{i}}^{(i)}\left(x_{i}\right), \mathbf{x} \in I_{a b}
$$

are called the basic functions of this partition.

The fuzzy partition of $I_{a b}$ is said to fulfill the Ruspini condition on some subset $I \subset I_{a b}$ if the basic functions form a partition of unity on $I$, i.e., $\sum_{\mathbf{j}} A_{\mathbf{j}}(\mathbf{x})=1$ for all $\mathbf{x} \in I$.

\subsection{Discrete $F^{m}$-transform}

Fix a FP $\mathbb{A}$ of $I_{a b}$. Let $\Delta=\left\{\mathbf{z}_{1}, \ldots, \mathbf{z}_{L}\right\} \subset I_{a b}$ be a discrete subset of the cuboid $I_{a b}$ and $f: \Delta \rightarrow \mathbb{R}$. Denote $y_{s}=f\left(\mathbf{z}_{s}\right)$, for each $s \in\{1, \ldots, L\}$, and let $\mathbf{Y}=\left(y_{1}, y_{2}, \ldots, y_{L}\right)^{T}$ be a column vector containing the values of the function $f$.

Fix any ordering of the set $\left\{\mathbf{l} \in \mathbb{N}_{0}^{d}: 0 \leq|\mathbf{l}| \leq m\right\}$ and suppose that it is given using the following notation: $\mathbf{l}_{r}, r \in\left\{1, \ldots, m^{\prime}\right\}$, where $m^{\prime}=\left(\begin{array}{c}m+d \\ d\end{array}\right)$ is the number of elements of this set.

For each $\mathbf{j}$ satisfying $0 \leq \mathbf{j} \leq \mathbf{N}$ construct the $L \times m^{\prime}$ matrix $\mathbf{X}_{\mathbf{j}}$ and the $L \times L$ matrix $\mathbf{A}_{\mathbf{j}}$ as follows:

$$
\begin{aligned}
& \mathbf{X}_{\mathbf{j}}=\left(\begin{array}{ccc}
P_{\mathbf{l}_{1}}\left(\mathbf{z}_{1}-\mathbf{t}_{\mathbf{j}}\right) & \ldots & P_{\mathbf{1}_{m^{\prime}}}\left(\mathbf{z}_{1}-\mathbf{t}_{\mathbf{j}}\right) \\
P_{\mathbf{l}_{1}}\left(\mathbf{z}_{2}-\mathbf{t}_{\mathbf{j}}\right) & \ldots & P_{\mathbf{l}_{m^{\prime}}}\left(\mathbf{z}_{2}-\mathbf{t}_{\mathbf{j}}\right) \\
& \ddots & \\
& \ldots & P_{\mathbf{l}_{m^{\prime}}}\left(\mathbf{z}_{L}-\mathbf{t}_{\mathbf{j}}\right)
\end{array}\right) \text {, } \\
& \mathbf{A}_{\mathbf{j}}=\operatorname{diag}\left(A_{\mathbf{j}}\left(\mathbf{z}_{1}\right), \ldots, A_{\mathbf{j}}\left(\mathbf{z}_{L}\right)\right) \text {, }
\end{aligned}
$$

where $P_{\mathbf{l}}(\mathbf{x})=\mathbf{x}^{\mathbf{l}}$ and $\mathbf{t}_{\mathbf{j}}=\left(t_{1, j_{1}}, t_{2, j_{2}}, \ldots, t_{d, j_{d}}\right)$.

Definition 2. The set $\Delta$ is sufficiently dense in the fuzzy partition $\mathbb{A}$ w.r.t. $m$ if for each $\mathbf{j}$ the matrix $\mathbf{X}_{\mathbf{j}}^{T} \mathbf{A}_{\mathbf{j}} \mathbf{X}_{\mathbf{j}}$ is invertible.

Notice that this definition slightly differs from [4, Def. $3]$.

Definition 3. Let $\mathbf{p}=\left(p_{\mathbf{j}}: 0 \leq \mathbf{j} \leq \mathbf{N}\right)$ be a collection of polynomials of the total degree at most $m$ in $d$ variables. Then the function

$$
F_{m}^{\leftarrow}[\mathbf{p}](\mathbf{t})=\frac{\sum_{\mathbf{j}} p_{\mathbf{j}}(\mathbf{t}) A_{\mathbf{j}}(\mathbf{t})}{\sum_{\mathbf{j}} A_{\mathbf{j}}(\mathbf{t})}
$$

is called the inverse $F^{m}$-transform of $\mathbf{p}$.

Definition 4. Let $\Delta=\left\{\mathbf{z}_{1}, \ldots, \mathbf{z}_{L}\right\} \subset I_{a b}$ be sufficiently dense in the FP $\mathbb{A}$ w.r.t. $m$ and let $f: \Delta \rightarrow \mathbb{R}$. Then the collection $F_{m}[f]=\left(F_{m, \mathbf{j}}: 0 \leq \mathbf{j} \leq \mathbf{N}\right)$ of polynomials $F_{m, \mathbf{j}}$ defined by

$$
F_{m, \mathbf{j}}^{\rightarrow}(\mathbf{t})=\sum_{r=1}^{m^{\prime}} \beta_{r}^{(\mathbf{j})} P_{\mathbf{l}_{r}}\left(\mathbf{t}-\mathbf{t}_{\mathbf{j}}\right),
$$

where

$$
\beta^{(\mathbf{j})}=\left(\mathbf{X}_{\mathbf{j}}^{T} \mathbf{A}_{\mathbf{j}} \mathbf{X}_{\mathbf{j}}\right)^{-1} \mathbf{X}_{\mathbf{j}}^{T} \mathbf{A}_{\mathbf{j}} \mathbf{Y},
$$

is said to be the direct discrete $F^{m}$-transform of $f$ w.r.t. the fuzzy partition $\mathbb{A}$.

The inverse $F^{m}$-transform of this collection, i.e., the function

$$
F_{m}[f](\mathbf{t})=\frac{\sum_{\mathbf{j} \in I_{\mathbf{N}}} F_{m, \mathbf{j}}^{\rightarrow}(\mathbf{t}) A_{\mathbf{j}}(\mathbf{t})}{\sum_{\mathbf{j} \in I_{\mathbf{N}}} A_{\mathbf{j}}(\mathbf{t})},
$$

is called the (composite) $F^{m}$-transform of $f$. 


\subsection{Fuzzy partition based on B-splines}

We form a FP in each dimension by using central Bsplines as the generating function. Central B-splines [15] are even B-splines that have 1-equidistant knots. For a fixed degree the central B-spline is unique (up to a constant factor). The properties of B-splines and construction of a fuzzy partition using central B-spline as the generating function are described in more details in $[7]$.

Definition 5. The central B-spline of degree $2 k-1$, denoted by $\phi_{2 k-1}$, is the unique piecewise polynomial function satisfying the following requirements: 1) for each $j \in\{-k, \ldots, k-1\}$ the restriction of $\phi_{2 k-1}$ to $[i, i+1]$ is a polynomial of degree at most $2 k-1 ; 2)$ $\left.\phi_{2 k-1} \in C^{2 k-2}(\mathbb{R}) ; 3\right) \phi_{2 k-1}(t)=0$ if $\left.t \notin(-k, k) ; 4\right)$ $\int_{\mathbb{R}} \phi_{2 k-1}(t) \mathrm{d} t=1$.

Fix $\mathbf{N}, \mathbf{k} \in \mathbb{N}^{d}$ such that $\mathbf{N} \geq 4 \mathbf{k}-1$. For all $i \in$ $\{1, \ldots, d\}$, let $A^{(i)}=\phi_{2 k_{i}-1}$. Denote $h_{i}=\left(b_{i}-a_{i}\right) / N_{i}$ and define $h_{i}$-equidistant nodes

$$
t_{i, j_{i}}=a_{i}+h_{i} j_{i}, \quad j_{i} \in\left\{0, \ldots, N_{i}\right\} .
$$

We also denote $\mathbf{t}_{\mathbf{j}}=\left(t_{1, j_{1}}, \ldots, t_{d, j_{d}}\right)$, for $0 \leq \mathbf{j} \leq \mathbf{N}$, called the basic nodes of this FP, and

$$
A_{j_{i}}^{(i)}\left(x_{i}\right)=\phi_{2 k_{i}-1}\left(\frac{x_{i}-t_{i, j_{i}}}{h_{i}}\right), \quad x_{i} \in\left[a_{i}, b_{i}\right],
$$

for all $j_{i} \in\left\{k_{i}, \ldots, N_{i}-k_{i}\right\}$ and $i \in\{1, \ldots, d\}$.

Then the basic functions $A_{k_{i}}^{(i)}, \ldots, A_{N_{i}-k_{i}}^{(i)}$ form a generalized $\left(h_{i}, h_{i} k_{i}\right)$-uniform fuzzy partition of $\left[a_{i}, b_{i}\right]$ (the different indexing allows to index the basic nodes from 0 to $\mathbf{N}$ ). We refer to the respective multivariate $\mathrm{FP}$ as $\operatorname{FPB}(\mathbf{k}, \mathbf{N})$. It can be shown that the described FP fulfills the Ruspini condition on $\prod_{i=1}^{d}\left[t_{i, 2 k_{i}-1}, t_{i, N_{i}-2 k_{i}+1}\right]$.

It is well-known that the composite $F^{m}$-transform is exact for polynomials of degree $\leq m$. The main advantage of the FPB is that the composite transform is exact for polynomials of degree $2 m+1$ (as long as it does not exceed the respective spline degree in each dimension) in the set where the Ruspini condition is fulfilled.

Let the discrete set $\Delta$ consists of the basic nodes $\mathbf{t}_{\mathbf{j}}$, $0 \leq \mathbf{j} \leq \mathbf{N}$ (this set is sufficiently dense w.r.t. the FP iff $m \leq 2 k_{i}-2$ for each $\left.i\right)^{1}$. Let $F_{m}[f]$ stand for the composite $F^{m}$-transform of $f: \Delta \rightarrow \mathbb{R}$ w.r.t. $\operatorname{FPB}(\mathbf{k}, \mathbf{N})$.

Theorem 6. Let non-negative integers $r, m$ satisfy

$$
r \leq \min \left\{2 m+1,2 k_{1}-1, \ldots, 2 k_{d}-1\right\}
$$

\footnotetext{
${ }^{1}$ One could allow larger values of $m$ by taking finer subgrids $\Delta, \tilde{\Delta}$, see [7] for details; for simplicity, we consider here only the case when $\tilde{\Delta}$ consists only of the basic nodes.
}

and $m \leq \min _{i}\left(2 k_{i}-2\right)$. Then for all $f \in \mathbb{P}_{r}^{d}$

$$
F_{m}[f](\mathbf{x})=f(\mathbf{x}) \quad \text { for all } \mathbf{x} \in \prod_{i=1}^{d}\left[t_{i, 2 k_{i}-1}, t_{i, N_{i}-2 k_{i}+1}\right]
$$

\section{Extended multivariate FPB and modified $F$-transform}

\subsection{Extended FPB}

Let $I_{a b}=\prod_{i=1}^{d}\left[a_{i}, b_{i}\right]$ be fixed, as well as appropriate parameters $\mathbf{k}, \mathbf{N} \in \mathbb{N}^{d}$ with $\mathbf{N} \geq 4 \mathbf{k}-1$. As previously, associate with $\mathbf{k}, \mathbf{N}$ points $\mathbf{t}_{\mathbf{j}} \in I_{a b}$ with $0 \leq \mathbf{j} \leq \mathbf{N}$. Define also the vector $\mathbf{h}$ with $\mathbf{h}=\left(h_{1}, \ldots, h_{d}\right)$, where $h_{i}=\frac{b_{i}-a_{i}}{N_{i}}$. To achieve an analog of Theorem 6 , we have to ensure that the Ruspini condition is satisfied in the whole domain. As in the $1 \mathrm{D}$ case, this is ensured by adding more basic functions to the fuzzy partition.

First we extend the given domain in the $i$-th dimension for each $i$ by the interval of length $\left(2 k_{i}-1\right) h_{i}$, to the left and right of $a_{i}, b_{i}$, respectively; denote the resulting set as

$$
I_{\widetilde{a b}}=\prod_{i=1}^{d}\left[a_{i}-\left(2 k_{i}-1\right) h_{i}, b_{i}+\left(2 k_{i}-1\right) h_{i}\right]
$$

Then construct the B-spline based fuzzy partition $\operatorname{FPB}(\mathbf{k}, \mathbf{N}+4 \mathbf{k}-2)$ of the set $I_{\widetilde{a b}}$, denoted by $\tilde{\mathbb{A}}$. The basic nodes of this fuzzy partition include the previous $\mathbf{t}_{\mathbf{j}}$ with $0 \leq \mathbf{j} \leq \mathbf{N}$, but there are now additional nodes outside the initial domain $I_{a b}$. All the basic nodes of $\tilde{\mathbb{A}}$ are labeled $\mathbf{t}_{\mathbf{j}}$ with $-(2 \mathbf{k}-1) \leq \mathbf{j} \leq \mathbf{N}+(2 \mathbf{k}-1)$ and the basic functions of $\tilde{\mathbb{A}}$ are $A_{\mathbf{j}}$ with multi-index $\mathbf{j}$ satisfying $-\mathbf{k}+1 \leq \mathbf{j} \leq \mathbf{N}+\mathbf{k}-1$.

By construction, the subset where the Ruspini condition holds for $\tilde{\mathbb{A}}$ is the initial domain $I_{a b}$. Therefore, when we restrict all basic functions $A_{\mathbf{j}}$ to $I_{a b}$, we obtain a fuzzy partition of $I_{a b}$, which fulfills the Ruspini condition everywhere in $I_{a b}$. We call this fuzzy partition the $(\mathbf{k}, \mathbf{N})$-eFPB of $I_{a b}$ and denote it by $\mathbb{A}$ :

$$
\mathbb{A}=\left\{A_{\mathbf{j}} I_{a b}:-\mathbf{k}+1 \leq \mathbf{j} \leq \mathbf{N}+\mathbf{k}-1\right\} .
$$

Furthermore, the discrete set $\Delta$ consists of all basic nodes in the initial domain $I_{a b}$, i.e.,

$$
\Delta=\left\{\mathbf{t}_{\mathbf{j}}: 0 \leq \mathbf{j} \leq \mathbf{N}\right\}
$$

We can only access functions in $C\left(I_{a b}\right)$ via their values on $\Delta$ (i.e., for $f \in C\left(I_{a b}\right)$ only the values $f\left(\mathbf{t}_{\mathbf{j}}\right)$ at the respective nodes are known). The set $\Delta$ can be naturally extended to the wider set

$$
\tilde{\Delta}=\left\{\mathbf{t}_{\mathbf{j}}:-(2 \mathbf{k}-1) \leq \mathbf{j} \leq \mathbf{N}+(2 \mathbf{k}-1)\right\} \subset I_{\widetilde{a b}} .
$$


If a function $f$ were defined at $\tilde{\Delta}$ (and we could access those values outside $I_{a b}$ ), we could perform the usual $F^{m}$-transform w.r.t. $\tilde{\mathbb{A}}$ on $I_{\widetilde{a b}}$ and due to Theorem 6 obtain good approximation of $f$ in $I_{a b}$. However, the function $f$ may not be even defined outside $I_{a b}$. So this approach fails, but it also serves as motivation for our strategy for achieving uniform approximation in the whole $I_{a b}$ : let us extrapolate function $f$ to some small vicinity outside $I_{a b}$ and then apply the usual $F^{m}$-transform w.r.t. $\tilde{\mathbb{A}}$ on $I_{\tilde{a} b}$. Since this is equivalent to modifying the way the $F^{m}$-transform components $F_{m, \mathbf{j}}$ are defined, with $\mathbf{j}$ corresponding to the fuzzy sets $A_{\mathbf{j}}$ near the boundary of $I_{a b}$, we refer to this method as the modified $F^{m}$-transform.

\subsection{Extrapolation operators}

For every $\epsilon>0$ define $S_{\epsilon}=\prod_{i=1}^{d}\left[a_{i}-\epsilon, b_{i}+\epsilon\right]$. Fix a nonnegative integer $M$ and a positive real $\delta$. Suppose that for every $\epsilon>0$ there are defined linear bounded operators $\mathfrak{E}_{\epsilon}: C\left(I_{a b}\right) \rightarrow C\left(S_{\epsilon}\right)$ such that the following holds:

1. $\mathfrak{E}_{\epsilon} f=f$ for every polynomial $f \in \mathbb{P}_{M}^{d}$;

2. $\left(\mathfrak{E}_{\epsilon} f\right)(\mathbf{x})=f(\mathbf{x})$ for all $\mathbf{x} \in I_{a b}$;

3. $\mathfrak{E}_{\epsilon}$ are $\delta$-local, i.e., whenever $f, g \in C\left(I_{a b}\right)$ coincide on $\mathfrak{B}_{\delta \epsilon}\left(\mathbf{x}_{0}\right) \cap I_{a b}$ for some $\mathbf{x}_{0} \in I_{a b}$, we also have

$$
\mathfrak{E}_{\epsilon} f(\mathbf{x})=\mathfrak{E}_{\epsilon} g(\mathbf{x}) \text { for all } \quad \mathbf{x} \in \mathfrak{B}_{\epsilon}\left(\mathbf{x}_{0}\right) \cap S_{\epsilon} ;
$$

4. the family $\left\{\mathfrak{E}_{\epsilon}\right\}_{\epsilon}$ is uniformly bounded, i.e., there exists a constant $C_{\star}>0$ (independent of $\epsilon$ ) such that $\left\|\mathfrak{E}_{\epsilon}\right\| \leq C_{\star}$ for all $\epsilon$.

We refer to the operator $\mathfrak{E}_{\epsilon}$ as a $\delta$-local order- $M$ extrapolator (for functions $f \in C\left(I_{a b}\right)$ ).

We note that such a family of extrapolators can be constructed via some local extrapolation technique, e.g., via moving least squares [1, Chapter 22] with a weight function ensuring that we use only the known values of the function in a certain vicinity near the point at which we intend to compute the extrapolated value.

\subsection{Modification of $F^{m}$-transform}

Let $\delta>1$ be a positive real; fix a family of $\delta$-local order- $M$ extrapolators $\left\{\mathfrak{E}_{\epsilon}\right\}_{\epsilon}$ for functions $f \in C\left(I_{a b}\right)$. Further, fix $\mathbf{k} \in \mathbb{N}^{d}$ and $\mathbf{N} \in \mathbb{N}^{d}$, s.t. $\mathbf{N} \geq 4 \mathbf{k}-1$. Let

- $\mathbb{A}$ be the corresponding $(\mathbf{k}, \mathbf{N})$-eFPB of $I_{a b}$;

- $I_{\widetilde{a b}}=\prod_{i=1}^{d}\left[a_{i}-\left(2 k_{i}-1\right) h_{i}, b_{i}+\left(2 k_{i}-1\right) h_{i}\right]$;
- $\tilde{\mathbb{A}}$ be the respective unrestricted FPB of the wider set $I_{\widetilde{a b}}$.

Set $\delta^{\prime}=2\|\mathbf{k}\|_{\infty}$ and $h=\|\mathbf{h}\|_{\infty}$. We shall use $\mathfrak{E}$ as a shorthand for $\mathfrak{E}_{\delta^{\prime} h}$. Notice that $h \delta^{\prime} \geq\left(2 k_{i}-1\right) h_{i}$ for each $i$, thus $\mathfrak{E} f \in C\left(I_{\widetilde{a b}}\right)$.

Let $\Delta$ and $\tilde{\Delta}$ be as described before; let $m$ be a nonnegative integer s.t. $m \leq 2 k_{i}-2$ for all $i$, so that $\tilde{\Delta}$ is sufficiently dense in $\tilde{\mathbb{A}}$. Then we call the direct $F^{m}$ transform (w.r.t the fuzzy partition $\tilde{\mathbb{A}}$ of $I_{\tilde{a b}}$ ) of the extrapolant $\mathfrak{E} f$ a modified $F^{m}$-transform:

Let $f \in C\left(I_{a b}\right)$ and $\mathfrak{E}$ be an extrapolator described above. Denote $\tilde{f}=\mathfrak{E} f$ and let

$$
F_{m}^{\rightarrow}[\tilde{f}]=\left(F_{m, \mathbf{j}}^{\rightarrow}[\tilde{f}]:-\mathbf{k} \leq \mathbf{j} \leq \mathbf{N}+\mathbf{k}\right)
$$

be the direct $F^{m}$-transform of $\tilde{f}$ w.r.t. $\tilde{\mathbb{A}}$.

We refer to the collection $F_{m}^{\rightarrow}[\tilde{f}]$ as the direct modified $F^{m}$-transform (shortened to direct $\tilde{F}^{m}$-transform) of $f$ (based on the extrapolants $\mathfrak{E}_{\epsilon}$, w.r.t. the fuzzy partition $\mathbb{A})$ and denote it by $\tilde{F}_{m}^{\rightarrow}[f]$. Similarly, the $\mathbf{j t h}$ component of $\tilde{F}_{m}[f]$ is called the $\mathbf{j t h}$ component of the direct $\tilde{F}^{m}$-transform of $f$ and denoted by $\tilde{F}_{m, \mathbf{j}}^{\rightarrow}[f]$.

The (composite) $\tilde{F}^{m}$-transform is defined as the usual inverse $F^{m}$-transform applied to the collection $\tilde{F}_{m}^{\rightarrow}[f]$ and denoted by $\tilde{F}_{m}[f]$.

Theorem 7. Under the constraints described previously, the following holds:

1. $\tilde{F}_{m}$ is a linear operator from $C\left(I_{a b}\right)$ to $C\left(I_{a b}\right)$;

2. $\tilde{F}_{m, \mathbf{j}}[f]=F_{m, \mathbf{j}}^{\rightarrow}[f]$ for all $\mathbf{j}$ s.t. $\mathbf{k} \leq \mathbf{j} \leq \mathbf{N}-\mathbf{k}$;

3. Suppose that $M \leq 2 m+1$. Let integer $r$ satisfy $0 \leq r \leq \min \left\{M, 2 k_{1}-1, \ldots, 2 k_{d}-1\right\}$. Then $p(\mathbf{x})=\tilde{F}_{m}[p](\mathbf{x})$ for all $p \in \mathbb{P}_{r}^{d}$ and $\mathbf{x} \in I_{a b}$.

Proof. The linearity of $\tilde{F}_{m}$ follows from that of $\mathfrak{E}$ and the usual $F^{m}$-transform. For the second claim, notice that the corresponding basic function $A_{\mathbf{j}}$ is supported in the product of intervals $\left(t_{i, j_{i}-k_{i}}, t_{i, j_{i}+k_{i}}\right) \subset\left[a_{i}, b_{i}\right]$, where $f \equiv \tilde{f}$. Finally, the modified transform is based on order- $M$ extrapolators and $p \in \mathbb{P}_{M}^{d}$, thus $\tilde{p} \equiv p$. Since $r \leq M \leq 2 m+1$ and $r \leq 2 k_{i}-1$, Theorem 6 implies that the composite transform of $p$ w.r.t the fuzzy partition $\tilde{\mathbb{A}}$ of $I_{\widetilde{a b}}$ coincides with $p$ on the subset where the Ruspini condition holds. By construction, this subset of $I_{\widetilde{a b}}$ equals the initial cuboid $I_{a b}$, thus $\tilde{F}_{m}[p] \equiv p$ on $I_{a b}$.

Now we note that the family of modified $F^{m}$ transforms (with fixed $I_{a b}$ and $\mathbf{k}$ but varying $\mathbf{N}$ and, thus, $h$ ) forms a family of uniformly bounded linear operators acting from $C\left(I_{a b}\right)$ to itself. 
Theorem 8. Let $\mathbf{k}, m \geq 0$ and a family of $\delta$-local order-M extrapolators $\left\{\mathfrak{E}_{\epsilon}\right\}_{\epsilon}$ be as described previously. Consider the family of operators $\left\{\tilde{F}_{m, \mathbf{N}}\right\}_{\mathbf{N}}$, where $\tilde{F}_{m, \mathbf{N}}: C\left(I_{a b}\right) \rightarrow C\left(I_{a b}\right)$ stands for the modified $F^{m}$-transform w.r.t. the $(\mathbf{k}, \mathbf{N})$-eFPB of $I_{a b}$, for all $\mathbf{N} \geq 4 \mathbf{k}-1$.

Then this family is uniformly bounded, i.e., there is a constant $C>0$, independent of $\mathbf{N}$, such that $\left|\tilde{F}_{m}[f]\right| \leq C\|f\|$ for all $f \in C\left(I_{a b}\right)$.

Sketch of the proof. The family of extrapolators $\left\{\mathfrak{E}_{\epsilon}\right\}_{\epsilon}$ is uniformly bounded by $C_{\star}$, thus for every $f \in C\left(I_{a b}\right)$ we have a bound on the extrapolated function's values: $|\tilde{f}(\mathbf{x})| \leq C_{\star}\|f\|$ for all $\mathbf{x} \in I_{\widetilde{a b}}$.

Therefore it suffices to show that the usual $F^{m_{-}}$ transform is uniformly bounded (when $\mathbf{k}$ is fixed, $\mathbf{N}$ varies, and w.r.t. the $\operatorname{FPB}(\mathbf{k}, \mathbf{N}+4 \mathbf{k}-2)$ of the set $I_{\widetilde{a b}}$, which itself depends on $\mathbf{N}$ ). In other words, it suffices to show that $F_{m}[g] \leq C^{\prime}\|g\|$ for all $g \in C\left(I_{\widetilde{a b}}\right)$, with $C^{\prime}$ independent of $h, \mathbf{N}$ or $g$; then the claim follows with $C=C^{\prime} C_{\star}$. The proof is somewhat technical and we omit it here, but it can be carried out similarly as the proof of a similar claim in [7, Lemma 9], by expressing the composite transform for each $\mathbf{x} \in I_{\widetilde{a b}}$ as

$$
F_{m}[g](\mathbf{x})=\sum_{\mathbf{j}} \sum_{\substack{\mathbf{l} \in \mathbb{N}_{0}^{d}: \\|\mathbf{l}| \leq m}} c_{\mathbf{j}, 1}[g] P_{\mathbf{j}, \mathbf{l}}(\mathbf{x}) A_{\mathbf{j}}(\mathbf{x})
$$

and showing that only $O(1)$ summands are nonzero, and among those summands each factor $P_{\mathbf{j}, \mathbf{l}}(\mathbf{x}) A_{\mathbf{j}}(\mathbf{x})$ is $O(1)$ and the remaining factor satisfies $c_{\mathbf{j}, 1}[g]=$ $O(\|g\|)$, with the big-O constants depending only on $\mathbf{k}, d$ and $m$.

Further we demonstrate the locality of $\tilde{F}_{m}$.

Theorem 9. Let $\mathbf{k}, \mathbf{N}, \delta \geq 1$ and an integer $m \geq 0$ be fixed as previously; denote $\bar{\delta}^{\prime}=2\|\mathbf{k}\|_{\infty}$ and $h=\|\mathbf{h}\|_{\infty}$. Fix a family of $\delta$-local order- $M$ extrapolators $\left\{\mathfrak{E}_{\epsilon}\right\}_{\epsilon}$ as described previously.

Suppose that $f, g \in C\left(I_{a b}\right)$ and $\mathbf{x}_{0} \in I_{a b}$ satisfy

$f(\mathbf{x})=g(\mathbf{x}) \quad$ for all $\quad \mathbf{x} \in I_{a b}$ s.t. $\left\|\mathbf{x}-\mathbf{x}_{0}\right\|_{\infty} \leq \delta \delta^{\prime} h$.

Then $\tilde{F}_{m}[f]\left(\mathbf{x}_{0}\right)=\tilde{F}_{m}[g]\left(\mathbf{x}_{0}\right)$.

Proof. We have $\tilde{F}_{m}[f](\mathbf{x})=F_{m}[\tilde{f}](\mathbf{x})$, where $F_{m}$ stands for the usual $F^{m}$-transform w.r.t. the fuzzy partition $\tilde{\mathbb{A}}$ of $I_{\widetilde{a b}}$ and $\tilde{f}=\mathfrak{E}_{h \delta^{\prime}} f$.

We shall show the following two assertions:

1. the functions $\tilde{f}$ and $\tilde{g}$ satisfy $\tilde{f}(\mathbf{x})=\tilde{g}(\mathbf{x})$ for all $\mathbf{x} \in I_{\widetilde{a b}}$ s.t. $\left\|\mathbf{x}-\mathbf{x}_{0}\right\|_{\infty} \leq \delta^{\prime} h$,
2. if the previous assertion holds, then the values $F_{m}[\tilde{f}]\left(\mathbf{x}_{0}\right)$ and $F_{m}[\tilde{g}]\left(\mathbf{x}_{0}\right)$ are equal.

Clearly, combining those two claims proves the theorem.

For the first assertion, notice that $f, g \in C\left(I_{a b}\right)$ coincide on $\mathfrak{B}_{\delta \delta^{\prime} h}\left(\mathbf{x}_{0}\right) \cap I_{a b}$. Since $\mathfrak{E}$ is $\delta$-local, this implies

$$
\tilde{f}(\mathbf{x})=\tilde{g}(\mathbf{x}) \quad \text { for all } \quad \mathbf{x} \in \mathfrak{B}_{\delta^{\prime} h}\left(\mathbf{x}_{0}\right) \cap S_{\delta^{\prime} h} .
$$

Since $S_{\delta^{\prime} h} \supset I_{\widetilde{a b}}$, we conclude the required $\tilde{f}(\mathbf{x})=\tilde{g}(\mathbf{x})$ for all $\mathbf{x} \in I_{\widetilde{a b}}$ s.t. $\left\|\mathbf{x}-\mathbf{x}_{0}\right\|_{\infty} \leq \delta^{\prime} h$.

To show the second claim, we note that there is a multi-index $\mathbf{j}^{\star}$ s.t.

$$
0 \leq j_{i}^{\star} \leq N_{i}-1 \quad \text { and } \quad t_{i, j_{i}^{\star}} \leq x_{0, i} \leq t_{i, j_{i}^{\star}+1} .
$$

Observe that $A_{\mathbf{j}}\left(\mathbf{x}_{0}\right)=0$ unless $\mathbf{j}^{\star}-\mathbf{k}+1 \leq \mathbf{j} \leq \mathbf{j}^{\star}+\mathbf{k}$. Let $I_{\star}$ stand for the set of all multi-indices $\mathbf{j}$ satisfying these constraints, i.e.,

$$
I_{\star}=\left\{\mathbf{j} \mid \mathbf{j}^{\star}-\mathbf{k}+1 \leq \mathbf{j} \leq \mathbf{j}^{\star}+\mathbf{k}\right\} .
$$

Then

$$
F_{m}[\tilde{f}]\left(\mathbf{x}_{0}\right)=\sum_{\mathbf{j} \in I_{\star}} F_{m, \mathbf{j}}^{\rightarrow}[\tilde{f}]\left(\mathbf{x}_{0}\right) A_{\mathbf{j}}\left(\mathbf{x}_{0}\right) .
$$

Obviously, $F_{m, \mathbf{j}}$ depends only of the function's values in the support on $A_{\mathbf{j}}$, i.e, in the set $\prod_{i=1}^{d}\left(t_{i, j_{i}-k_{i}}, t_{i, j_{i}+k_{i}}\right)$. Each $\mathbf{x}$ from this set satisfies

$$
\begin{aligned}
& x_{i} \geq t_{i, j_{i}-k_{i}} \geq t_{i, j_{i}^{\star}-2 k_{i}+1} \geq x_{0, i}-2 k_{i} h_{i}, \\
& x_{i} \leq t_{i, j_{i}+k_{i}} \leq t_{i, j_{i}^{\star}+2 k_{i}} \leq x_{0, i}+2 k_{i} h_{i},
\end{aligned}
$$

for all $i \in\{1, \ldots, d\}$. Since

$$
2 k_{i} h_{i} \leq \max _{i}\left(2 k_{i}\right) \cdot \max _{i} h_{i}=\delta^{\prime} h,
$$

we conclude that $\tilde{f} \equiv \tilde{g}$ on $\operatorname{supp} A_{\mathbf{j}}$ for all $\mathbf{j} \in I_{\star}$, hence $F_{m}[\tilde{f}]\left(\mathbf{x}_{0}\right)=F_{m}[\tilde{g}]\left(\mathbf{x}_{0}\right)$ as desired.

The previous two theorems imply the following:

Corollary 10. Let $\mathbf{k}, m \geq 0, \delta \geq 1$ and a family of $\delta$-local order- $M$ extrapolators $\left\{\mathfrak{E}_{\epsilon}\right\}_{\epsilon}$ be fixed as previously.

Suppose that there is a constant $K_{1}>0$ and an integer $n \geq 1$, such that for some $\mathbf{x}_{0} \in I_{a b}$ and all $h>0$ there is a function $f \in C\left(I_{a b}\right)$, possibly depending on $h$ and $\mathbf{x}_{0}$, which satisfies

$$
|f(\mathbf{x})| \leq K_{1} h^{n} \text { for all } \mathbf{x} \in \mathfrak{B}_{2 h \delta \delta^{\prime}}\left(\mathbf{x}_{0}\right) \cap I_{a b} .
$$

Then there is a constant $K_{2}>0$, independent of $h$ or $\mathbf{x}_{0}$, such that

$$
\left|\tilde{F}_{m}[f](\mathbf{x})\right| \leq K_{2} h^{n} \text { for all } \mathbf{x} \in \mathfrak{B}_{h \delta \delta^{\prime}}\left(\mathbf{x}_{0}\right) \cap I_{a b} .
$$


Proof. For the sake of simplicity, denote $r=h \delta \delta^{\prime}$. Fix any $\mathbf{x} \in \mathfrak{B}_{r}\left(\mathbf{x}_{0}\right) \cap I_{a b}$ and form a new function $f_{r} \in$ $C\left(I_{a b}\right)$ defined as $f_{r}(\mathbf{z})=f\left(g_{r}(\mathbf{z})\right)$, where $g_{r} \in C\left(I_{a b}\right)$ stands for

$$
g_{r}(\mathbf{z})=\mathbf{x}+(\mathbf{z}-\mathbf{x}) \cdot \min \left\{\|\mathbf{z}-\mathbf{x}\|_{\infty}, r\right\} .
$$

In other words, $g_{r}(\mathbf{z})$ is simply $\mathbf{z}$ if $\mathbf{z}$ is in the $\ell^{\infty}$-ball $\mathfrak{B}_{r}(\mathbf{x})$, otherwise it is the point on the boundary of this ball, which is on the segment joining $\mathbf{x}$ and $\mathbf{z}$.

By construction, $f_{r}$ values cannot exceed those of $f(\mathbf{z})$ when $\|\mathbf{z}-\mathbf{x}\|_{\infty} \leq r$. However, for each such $\mathbf{z}$ we have $\left\|\mathbf{z}-\mathbf{x}_{0}\right\| \leq\|\mathbf{z}-\mathbf{x}\|+\left\|\mathbf{x}-\mathbf{x}_{0}\right\| \leq 2 r$, thus

$$
|f(\mathbf{z})| \leq K_{1} h^{n} \quad \text { and } \quad\left\|f_{r}\right\| \leq K_{1} h^{n} .
$$

By construction $f_{r}$ and $f$ coincide on $\mathfrak{B}_{r}(\mathbf{x})$ with $r=$ $h \delta \delta^{\prime}$, thus Theorem 9 implies

$$
\left|\tilde{F}_{m}[f](\mathbf{x})\right|=\left|\tilde{F}_{m}\left[f_{r}\right](\mathbf{x})\right| .
$$

By Theorem $8,\left\|\tilde{F}_{m}\right\| \leq C$ for some constant $C$, independent of $h$, which implies

$$
\left|\tilde{F}_{m}[f](\mathbf{x})\right|=\left|\tilde{F}_{m}\left[f_{r}\right](\mathbf{x})\right| \leq C\left\|f_{r}\right\| \leq K_{1} C h^{n} .
$$

Since $K_{1}$ and $C$ are independent of $\mathbf{x}$ or $h$, we have the desired result with $K_{2}=C K_{1}$.

\section{Approximation of smooth functions}

Throughout this section, we fix

- $\mathbf{k} \in \mathbb{N}^{d}$;

- a real number $\delta \geq 1$;

- $\mathbf{N} \in \mathbb{N}^{d}$ satisfying $\mathbf{N} \geq 4 \mathbf{k}-1$;

- $0 \leq m \leq \min \left\{2 k_{1}-2 \ldots, 2 k_{d}-2\right\}$;

- a family of $\delta$-local order- $(2 m+1)$ extrapolators $\left\{\mathfrak{E}_{\epsilon}\right\}_{\epsilon}$ as described previously;

- the $(\mathbf{k}, \mathbf{N})$-eFPB of $I_{a b}$, denoted by $\mathbb{A}$.

Furthermore, $\tilde{F}_{m}$ stands for the modified $F^{m}$ transform w.r.t. the eFPB $\mathbb{A}$, based on the extrapolators $\left\{\mathfrak{E}_{\epsilon}\right\}_{\epsilon}$. We also denote

$$
h=\|\mathbf{h}\|_{\infty}=\max _{i} h_{i} \quad \text { and } \quad \delta^{\prime}=2\|\mathbf{k}\|_{\infty}=2 \max _{i} k_{i} .
$$

Under those assumptions the following holds:

Theorem 11. Let $r \in \mathbb{Z}$ satisfy

$$
0 \leq r \leq \min \left\{2 m+1,2 k_{1}-1, \ldots, 2 k_{d}-1\right\} .
$$

Then

$$
\left\|f-\tilde{F}_{m}[f]\right\|=O\left(h^{r+1}\right) \quad \text { for all } \quad f \in C^{r+1}\left(I_{a b}\right) .
$$

Proof. Fix any $f \in C^{r+1}\left(I_{a b}\right)$ and $\mathbf{x} \in I_{a b}$. Then there is a multi-index j s.t. $0 \leq j_{i} \leq N_{i}-1$ and

$$
t_{i, \mathbf{j}_{i}} \leq x_{i} \leq t_{i, \mathbf{j}_{i}+1}, i=1,2, \ldots, d \text {. }
$$

We take $\mathbf{x}_{0}:=\mathbf{t}_{\mathbf{j}}$ and apply the Taylor formula to $f$ with the Lagrange form of the remainder (note that $\left.\left\|\mathbf{x}-\mathbf{x}_{0}\right\|_{\infty} \leq h\right)$, obtaining

$$
f(\mathbf{x})=p_{r}(\mathbf{x})+\sum_{\substack{\beta: \\|\beta|=r+1}} \partial^{\beta} f\left(\mathbf{x}_{0}+\theta\left(\mathbf{x}-\mathbf{x}_{0}\right)\right) \frac{\left(\mathbf{x}-\mathbf{x}_{0}\right)^{\beta}}{\beta !},
$$

where $p_{r}$ is the Taylor polynomial for $f, \operatorname{deg} p_{r} \leq r$, $\mathbf{x}_{0}$ is the point of expansion, $\theta \in[0,1]$. Let us denote

$$
R_{s, \theta}(\mathbf{x}):=\sum_{\substack{\beta: \\|\beta|=s}} \partial^{\beta} f\left(\mathbf{x}_{0}+\theta\left(\mathbf{x}-\mathbf{x}_{0}\right)\right) \frac{\left(\mathbf{x}-\mathbf{x}_{0}\right)^{\beta}}{\beta !},
$$

then we can succinctly write $f(\mathbf{x})=p_{r}(\mathbf{x})+R_{r+1, \theta}(\mathbf{x})$. Notice that $R_{r+1, \theta}$ is a continuous function, since the respective derivatives of $f$ are. By the linearity of the $\tilde{F}^{m}$-transform,

$$
\tilde{F}_{m}[f]-f=\tilde{F}_{m}\left[R_{r+1, \theta}\right]-R_{r+1, \theta},
$$

since $\tilde{F}_{m}\left[p_{r}\right]=p_{r}$ by Theorem 7 .

Next we shall show that $R_{r+1, \theta}$ fulfills the requirements of Corollary 10, i.e., there is a positive constant $K_{1}$, independent of $\mathbf{x}, \mathbf{j}$ or $h$, such that

$$
\left|R_{r+1, \theta}(\mathbf{z})\right| \leq K_{1} h^{r+1} \quad \text { for all } \quad \mathbf{z} \in \mathfrak{B}_{2 h \delta \delta^{\prime}}\left(\mathbf{x}_{0}\right) \cap I_{a b} .
$$

To see that, choose any $\mathbf{z} \in I_{a b}$ s.t. $\left\|\mathbf{z}-\mathbf{x}_{0}\right\| \leq 2 h \delta \delta^{\prime}$. Since each $\partial^{\beta} f$ is continuous on the compact set $I_{a b}$, it has finite norm $\left\|\partial^{\beta} f\right\|$. Then

$$
\left|R_{r+1, \theta}(\mathbf{z})\right| \leq \sum_{\substack{\beta: \\|\beta|=r+1}} \frac{\left\|\partial^{\beta} f\right\|}{\beta !} \cdot\left|\left(\mathbf{z}-\mathbf{x}_{0}\right)^{\beta}\right| .
$$

Each component of $\mathbf{z}-\mathbf{x}_{0}$ is bounded by $2 h \delta \delta^{\prime}$, thus

$$
\left|\left(\mathbf{z}-\mathbf{x}_{0}\right)^{\beta}\right| \leq \prod_{i=1}^{d}\left(2 h \delta \delta^{\prime}\right)^{\beta_{i}}=\left(2 h \delta \delta^{\prime}\right)^{|\beta|}=\left(2 h \delta \delta^{\prime}\right)^{r+1} .
$$

Therefore $\left|R_{r+1, \theta}(\mathbf{z})\right| \leq K_{1} h^{r+1}$, where the constant

$$
K_{1}:=\left(2 \delta \delta^{\prime}\right)^{r+1} \sum_{|\beta|=r+1} \frac{\left\|\partial^{\beta} f\right\|}{\beta !}
$$

is independent of $h$ or $\mathbf{x}_{0}$. Now Corollary 10 implies that there is a positive constant $K_{2}$, independent of $\mathbf{x}_{0}$ or $h$, s.t.

$\left|\tilde{F}_{m}\left[R_{r+1, \theta}\right](\mathbf{z})\right| \leq K_{2} h^{r+1}$ for all $\mathbf{z} \in \mathfrak{B}_{h \delta \delta^{\prime}}\left(\mathbf{x}_{0}\right) \cap I_{a b}$. 
Since $\left|\mathbf{x}-\mathbf{x}_{0}\right| \leq h \leq h \delta \delta^{\prime}$, we conclude that also

$\left|R_{r+1, \theta}(\mathbf{x})\right| \leq K_{1} h^{r+1}$ and $\left|\tilde{F}_{m}\left[R_{r+1, \theta}\right](\mathbf{x})\right| \leq K_{2} h^{r+1}$.

Now these estimates together with (3) give

$$
\left|\tilde{F}_{m}[f](\mathbf{x})-f(\mathbf{x})\right| \leq\left(K_{1}+K_{2}\right) h^{r+1} .
$$

Since this holds for all $\mathbf{x} \in I_{a b}$ and $K_{1}, K_{2}$ are independent of $\mathbf{x}$ or $h$, we have obtained the desired uniform approximation of order $h^{r+1}$.

We illustrate the preceding theorem by illustrating approximation of two bivariate functions. We will set the same spline degree in each dimension, as well as the same number of basic nodes in each dimension, i.e., $\mathbf{k}=(k, k)$ and $\mathbf{N}=(N, N)$ for some valid $k, N$. The modified transform will be based on order- $(2 m+1)$ extrapolators, obtained via moving least squares approximation.

Let $f \in C^{\infty}\left([0,1]^{2}\right)$ be Franke's function [2], defined by

$$
\begin{aligned}
f(x, y) & =0.75 \mathrm{e}^{-1 / 4\left((9 x-2)^{2}+(9 y-2)^{2}\right)} \\
& +0.75 \mathrm{e}^{-1 / 49(9 x+1)^{2}-1 / 10(9 y-2)^{2}} \\
& +0.5 \mathrm{e}^{-1 / 4\left((9 x-7)^{2}+(9 y-3)^{2}\right)} \\
& -0.2 \mathrm{e}^{-(9 x-4)^{2}-(9 y-7)^{2}},
\end{aligned}
$$

illustrated in Figure 1. In Table 1 we show the approx-

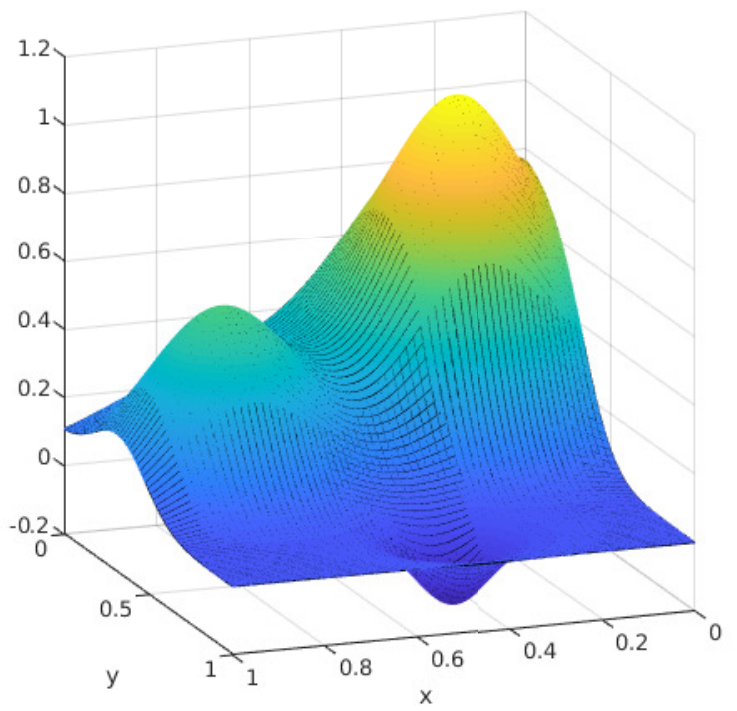

Figure 1: Franke's function

imation error of Franke's function when $k=2$ (i.e., the cubic B-splines in each dimension are used) and $m=1$, as well as when $k=3$ (the quintic B-splines in each dimension are used) and $m=2$. In the former case Theorem 11 predicts uniform approximation order $O\left(N^{-4}\right)$, in the latter case we expect approximation of order $O\left(N^{-6}\right)$. These estimates are consistent with the numeric approximation errors (obtained by splitting each basic interval in each dimension into 10 parts and obtaining a refinement of $\Delta$, then computing the error at each of the new nodes and taking the maximum absolute error) in Table 1. Furthermore, in Figure 2 we illustrate the approximation error when $k=2, m=1$ (i.e., the difference between Franke's function and its modified $F^{1}$-transform).

\begin{tabular}{c|cc}
\hline$N$ & $k=2, m=1$ & $k=3, m=2$ \\
\hline 64 & $2.745161 \mathrm{e}-04$ & $1.524311 \mathrm{e}-04$ \\
128 & $1.758060 \mathrm{e}-05$ & $4.600762 \mathrm{e}-06$ \\
256 & $1.014198 \mathrm{e}-06$ & $7.311263 \mathrm{e}-08$ \\
512 & $5.966349 \mathrm{e}-08$ & $1.076466 \mathrm{e}-09$ \\
1024 & $3.601380 \mathrm{e}-09$ & $1.607425 \mathrm{e}-11$ \\
2048 & $2.209334 \mathrm{e}-10$ & $3.154699 \mathrm{e}-12$ \\
\hline
\end{tabular}

Table 1: Maximum absolute error when approximating Franke's function

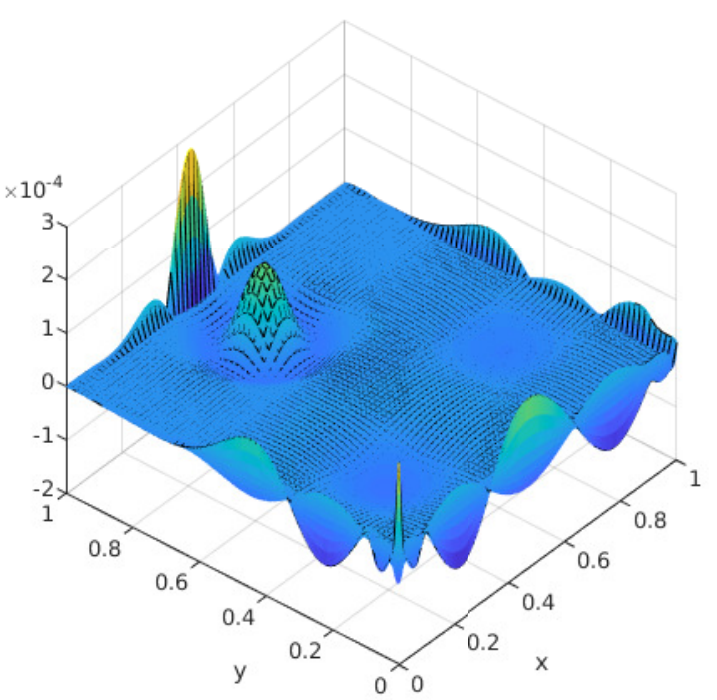

Figure 2: Approximation error of Franke's function by the modified $F^{1}$-transform, $\mathbf{k}=(2,2), \mathbf{N}=(64,64)$

The other function we approximate with the modified $F^{m}$-transform is $g \in C\left([-1,1]^{2}\right)$, defined by $g(x, y)=\sin \left(2 \pi^{2} x y\right) \exp \left(x^{2}+y^{2}\right)$. As previously, we consider approximation of $g$ with the modified $F^{1}$ transform when the fuzzy partition is formed by bicubic B-splines, and with the modified $F^{2}$-transform when the fuzzy partition is formed by biquintic Bsplines. Then the numeric approximation errors are 
computed (as described previously) and summarized in Table 2. Again, the results are consistent with the theoretic estimates $O\left(N^{-4}\right)$ and $O\left(N^{-6}\right)$.

\begin{tabular}{c|cc}
\hline$N$ & $k=2, m=1$ & $k=3, m=2$ \\
\hline 64 & $1.674709 \mathrm{e}+00$ & $2.119694 \mathrm{e}+00$ \\
128 & $3.063849 \mathrm{e}-01$ & $5.454578 \mathrm{e}-02$ \\
256 & $1.156863 \mathrm{e}-02$ & $3.651562 \mathrm{e}-03$ \\
512 & $6.291720 \mathrm{e}-04$ & $5.158642 \mathrm{e}-05$ \\
1024 & $3.930254 \mathrm{e}-05$ & $5.401694 \mathrm{e}-07$ \\
2048 & $2.449533 \mathrm{e}-06$ & $7.444174 \mathrm{e}-09$ \\
\hline
\end{tabular}

Table 2: Maximum absolute error when approximating $g(x, y)=\sin \left(2 \pi^{2} x y\right) \exp \left(x^{2}+y^{2}\right)$

\section{Conclusion}

Taking into account that a large part of applications of fuzzy transforms corresponds to the multi-dimensional case, in this paper we generalized the technique of modified multivariate $F^{m}$-transform with respect to fuzzy partition given by multivariate central B-splines of odd degree for each variable. Our future research will focus on applications of the modified spline-based $F^{m}$-transform for further development of numerical methods for solving differential equations.

\section{References}

[1] G. F. Fasshauer, Meshfree Approximation Methods with MATLAB, World Scientific Publishing Co., Inc., River Edge, NJ, USA, 2007.

[2] R. Franke, A critical comparison of some methods for interpolation of scattered data, Naval Postgraduate School Tech.Rep. NPS-53-79-003 (1979).

[3] P. Hodáková, Fuzzy (F-) transform of functions of two variables and its applications in image processing, Ph.D. thesis, University of Ostrava (2014).

[4] M. Holčapek, T. Tichý, Discrete fuzzy transform of higher degree, in: IEEE International Conference on Fuzzy Systems, FUZZ-IEEE 2014, Beijing, China, July 6-11, 2014, IEEE, 2014, pp. 604-611.

[5] M. Holčapek, T. Tichý, Discrete multivariate Ftransform of higher degree, in: IEEE International Conference on Fuzzy Systems, FUZZ-IEEE 2015, Istanbul, Turkey, August 2-5, 2015, IEEE, 2015 .
[6] M. Kokainis, S. Asmuss, Approximation properties of higher degree F-transforms based on B-splines, in: IEEE International Conference on Fuzzy Systems, FUZZ-IEEE 2015, Istanbul, Turkey, August 2-5, 2015, IEEE, 2015.

[7] M. Kokainis, S. Asmuss, Approximation by multivariate higher degree F-transform based on Bsplines, Soft Computing 21 (13) (2017) 35873614.

[8] M. Kokainis, S. Asmuss, Continuous and discrete higher-degree F-transforms based on B-splines, Soft Computing 21 (13) (2017) 3615-3639.

[9] M. Kokainis, S. Asmuss, Modified F-transform Based on B-splines, in: Information Processing and Management of Uncertainty in KnowledgeBased Systems. Theory and Foundations. 17th International Conference, IPMU 2018, Cádiz, Spain, June 11-15, 2018, Springer, Cham, 2018, pp. $175-186$.

[10] I. Perfilieva, Fuzzy transforms: Theory and applications, Fuzzy Sets and Systems 157 (8) (2006) 993-1023.

[11] I. Perfilieva, F-Transform, in: J. Kacprzyk, W. Pedrycz (Eds.), Springer Handbook of Computational Intelligence, Springer, Berlin, Heidelberg, 2015, pp. 113-130.

[12] I. Perfilieva, M. Dan̆ková, B. Bede, Towards a higher degree F-transform, Fuzzy Sets and Systems 180 (1) (2011) 3-19.

[13] I. Perfilieva, E. Haldeeva, Fuzzy transformation and its applications, in: Proceedings of the 4th Czech-Japan Seminar on Data Analysis and Decision Making under Uncertainty, Czech Republic, 2001, pp. 116-124.

[14] I. Perfilieva, P. Hodáková, $F^{1}$-transform of functions of two variables, in: J. Montero, G. Pasi, D. Ciucci (Eds.), Proceedings of EUSFLAT 2013, Milan, Italy, Vol. 32 of Advances in Intelligent Systems Research, Atlantis Press, 2013, pp. 547553.

[15] I. J. Schoenberg, Cardinal Spline Interpolation, Vol. 12 of CBMS, SIAM, 1973.

[16] M. Štěpnička, R. Valašek, Fuzzy transforms for functions with two variables, in: Proceedings of the 6th Czech-Japan Seminar on Data Analysis and Decision Making under Uncertainity, 2013, pp. 100-107. 